\title{
FIBRATIONS WITH CONSTANT SCALAR CURVATURE KÄHLER METRICS AND THE CM-LINE BUNDLE
}

\author{
Joel Fine
}

\begin{abstract}
Let $\pi: X \rightarrow B$ be a holomorphic submersion between compact Kähler manifolds of any dimensions, whose fibres and base have no non-zero holomorphic vector fields and whose fibres admit constant scalar curvature Kähler metrics. This article gives a sufficient topological condition for the existence of a constant scalar curvature Kähler metric on $X$. The condition involves the CM-line bundle - a certain natural line bundle on $B$-which is proved to be nef. Knowing this, the condition is then implied by $c_{1}(B)<0$. This provides infinitely many Kähler manifolds of constant scalar curvature in every dimension, each with Kähler class arbitrarily far from the canonical class.
\end{abstract}

\section{Introduction}

1.1. Background. In [4] Calabi proposed that, when one exists, a constant scalar curvature Kähler ( $\operatorname{cscK})$ metric should provide a canonical representative for a given Kähler class. Since this suggestion, much work has focused on the topic. The general existence theory has been looked at in depth, motivated by a suggestion of Yau [21] relating the existence of a Kähler-Einstein metric on a Fano manifold to the stability of the canonical polarisation in the sense of geometric invariant theory. Later, Tian [18] introduced the notion of K-stability, and suggested that the existence of a cscK metric in the first Chern class of an ample line bundle $L$ should be equivalent to the Kstability of the polarisation defined by $L$. Finally, Donaldson [6] gave an interpretation of the conjecture in terms of symplectic geometry and moment maps. See [8] for a precise statment of the conjecture, including a definition of K-stability.

The difficulty, from the analytic viewpoint, in determining whether or not a cscK metric exists is that the resulting PDE is fourth order and fully non-linear. Despite this, some examples have been found, but only in situations with prescribed geometry which is then used get a handle on the PDE. For two recent such examples see $[1,17]$.

This paper adds to the list of examples, the prescribed geometry here being that of a fibration. CscK metrics are found on the total space of a holomorphic submersion $X \rightarrow B$ between compact Kähler manifolds. Even in this restricted context, however, a clear relationship between the existence of a cscK metric and stability arises. Moreover, the varying moduli of the fibres plays an important rôle. One is led to a natural line bundle on $B$ - the so-called CM-line bundle - which is shown to be nef on the moduli space of $\mathrm{K}$-semi-stable polarised varieties (ignoring the technical issues of whether such a space exists).

Received by the editors March 4, 2006. 
1.2. Overview of the results. Let $\pi: X \rightarrow B$ be a holomorphic submersion between compact Kähler manifolds and let $L \rightarrow X$ be a relatively ample line bundle. There is a natural line bundle, called the CM-line bundle, on $B$ which was introduced by Tian [19]. It is defined as follows.

Let $Y$ be a fibre, $n=\operatorname{dim} Y$ and let $s$ denote the constant

$$
s=\frac{\left.n c_{1}(Y) c_{1}(L)\right|_{Y} ^{n-1}}{\left.c_{1}(L)\right|_{Y} ^{n}}
$$

(In terms of differential geometry, given a Kähler metric in $c_{1}(L)$, its restriction to a fibre has average scalar curvature $2 \pi s$. In terms of algebraic geometry, $s$ is essentially the second coefficient in the Hilbert polynomial of $\left.L\right|_{Y}$.) Let $K_{X / B}$ denote the relative canonical bundle, and let $\mathcal{E}$ denote the virtual bundle

$$
\mathcal{E}=(n+1)\left(K_{X / B}^{*}-K_{X / B}\right) \otimes\left(L-L^{*}\right)^{n}-s\left(L-L^{*}\right)^{n+1} .
$$

The CM-line bundle is then $L_{\mathrm{CM}}=\left[\operatorname{det}\left(\pi_{!} E\right)\right]^{*}$. The bundle makes sense when $\pi$ is a flat proper morphism of varieties. This level of generality is not needed for the result concerning existence of cscK metrics.

In fact, only the first Chern class of $L_{\mathrm{CM}}$ is used in this article. It can be defined without recourse to virtual bundles or direct images: by Grothendieck-RiemannRoch,

$$
c_{1}\left(L_{\mathrm{CM}}\right)=2^{n+1} \pi_{*}\left[\left((n+1) c_{1}\left(K_{X / B}\right)+s c_{1}(L)\right) c_{1}(L)^{n}\right] .
$$

Notice that $c_{1}\left(L_{\mathrm{CM}}\right)$ is unchanged when $L$ is twisted by a line bundle pulled back from $B$. This is because after pushing forward the factor involving $s$ cancels other unwanted terms.

The relation of $L_{\mathrm{CM}}$ to K-stability and the existence of $\csc \mathrm{K}$ metrics has already been studied by Tian, [18] and Paul-Tian $[16,15]$. The fact that $c_{1}\left(L_{\mathrm{CM}}\right)$ is well behaved when all fibres of $X$ admit a cscK metric is noted in Fujiki-Schumacher [10].

For notational convenience, let $\alpha$ denote the following class in $H^{1,1}(B)$ :

$$
\alpha=\frac{c_{1}\left(L_{\mathrm{CM}}\right)}{2^{n+1}(n+1) \pi_{*}\left(c_{1}\left(L^{n}\right)\right)} .
$$

The main theorem proved here is the following. (See section 2 for the proof.)

Theorem 1.1. Let $\pi: X \rightarrow B$ be a holomorphic submersion between compact Kähler manifolds whose fibres and base admit no non-zero holomorphic vector fields. Let $L$ be a relatively ample line bundle on $X$ such that the restriction of $c_{1}(L)$ to each fibre admits a cscK metric. Suppose, moreover, that $\alpha-c_{1}(B) \geq 0$.

Then, for all sufficiently large $r$, the class

$$
\kappa_{r}=c_{1}(L)+r \pi^{*} \kappa_{B}
$$

contains a cscK metric where, if $\alpha-c_{1}(B)=0, \kappa_{B}$ is any Kähler class on the base, whilst if $\alpha-c_{1}(B)>0$, then $\kappa_{B}=\alpha-c_{1}(B)$.

In checking the condition $\alpha-c_{1}(B) \geq 0$, it is helpful to know about the positivity of $\alpha$ itself. This is provided by the following result. (See section 3 for the proof.) Here, a line bundle $E \rightarrow Y$ is asymptotically Hilbert stable if for all sufficiently large $m, E^{m}$ gives a Hilbert stable embedding of $Y$. 
Theorem 1.2. Let $\pi: X \rightarrow B$ be a flat proper morphism of varieties. Let $L$ be a relatively ample line bundle on $X$ whose restriction to a generic fibre is asymptotically Hilbert semi-stable.

Then $L_{\mathrm{CM}}$ is nef; that is, $c_{1}\left(L_{\mathrm{CM}}\right)$ evaluates non-negatively over any curve in $B$.

It is a result due to Donaldson [7] that if $E \rightarrow Y$ is an ample line bundle over a compact Kähler manifold with Aut $(Y, E)$ discrete (modulo scalars), then the existence of a cscK metric in $c_{1}(E)$ implies that $E$ is asymptotically Hilbert stable (and hence semi-stable). Combining this with Theorems 1.1 and 1.2 and the fact that nef plus positive is positive gives the following corollary.

Corollary 1.3. Let $\pi: X \rightarrow B$ be a holomorphic submersion of compact Kähler manifolds whose fibres and base admit no non-zero holomorphic vector fields. Let $L$ be a relatively ample line bundle on $X$ such that the restriction of $c_{1}(L)$ to each fibre admits a cscK metric. Suppose, moreover, that $c_{1}(B)<0$.

Then, for all sufficiently large $r$, the class

$$
\kappa_{r}=c_{1}(L)+r \pi^{*}\left(\alpha-c_{1}(B)\right)
$$

contains a cscK metric.

The condition $c_{1}(B)<0$ is straight forward to check and general enough to provide a large number of examples of cscK metrics. Some of these are described in section 4, giving infinitely many Kähler manifolds of constant scalar curvature in each dimension, all with Kähler class arbitrarily far from the canonical class.

When $c_{1}\left(L_{\mathrm{CM}}\right)$ is actually ample, one can say something in the case when $c_{1}(B)=$ 0 . In this direction, Fujiki-Schumacher [10] prove the following.

Theorem 1.4 (Fujiki-Schumacher). Let $\pi: X \rightarrow B$ be a holomorphic submersion whose fibres admit no non-zero holomorphic vector fields. Let $L \rightarrow X$ be a relatively ample line bundle such that the restriction of $c_{1}(L)$ to each fibre admits a cscK metrics. Suppose, moreover, that $\pi$ is not trivial over any curve in $B$. Then $c_{1}\left(L_{\mathrm{CM}}\right)$ is ample on $B$.

(Here $\pi$ is trivial over a curve means that all fibres of $\pi$ over that curve are biholomorhpic as polarised varieties.) Combining this with Theorem 1.1 gives the following.

Corollary 1.5. Let $\pi: X \rightarrow B$ be a holomorphic submersion of compact Kähler manifolds whose fibres and base admit no non-zero holomorphic vector fields. Let $L$ be a relatively ample line bundle on $X$ such that the restriction of $c_{1}(L)$ to each fibre admits a cscK metric. Suppose, moreover, that $\pi$ is not trivial on any curve in $B$ and that $c_{1}(B)=0$.

Then, for all sufficiently large $r$, the class

$$
\kappa_{r}=c_{1}(L)+r \pi^{*} \alpha
$$

contains a cscK metric.

Note that neither of Theorems 1.2 and 1.4 imply the other; whilst ample is stronger than nef, Theorem 1.2 makes no mention of cscK metrics and applies both when the fibres are singular and have automorhpisms. 
1.3. Acknowledgements. I would like to thank Simon Donaldson, Julius Ross and Richard Thomas for helpful conversations concerning this paper and related matters. I am also grateful to Bill Harvey for bringing to my attention the iterated surface bundles in section 4 .

\section{Proof of Theorem 1.1}

Assume throughout this section that $\pi: X \rightarrow B$ is a holomorphic submersion between compact Kähler manifolds, that the fibres and base of $\pi$ have no non-zero holomorphic vector fields, that $L$ is relatively ample and that the restriction of $c_{1}(L)$ to each fibre admits a cscK metric. Moreover, assume that $\alpha-c_{1}(B) \geq 0$. If $\alpha-c_{1}(B)=$ 0 , let $\kappa_{B}$ denote any Kähler class on $B$; if $\alpha-c_{1}(B)>0$, let $\kappa_{B}=\alpha-c_{1}(B)$.

For sufficiently large $r, \kappa_{r}=c_{1}(L)+r \pi^{*} \kappa_{B}$ is a Kähler class on $X$. The first step is to find a Kähler representative whose fibrewise restriction is cscK. Begin with any Kähler form $\omega^{\prime} \in \kappa_{r_{0}}$ for some large fixed $r_{0}$.

For $b \in B$, write $Y_{b}=\pi^{-1}(b)$ and let $\omega_{b}$ be a cscK metric in $\left.c_{1}(L)\right|_{Y_{b}}$. Since $H^{0}\left(T Y_{b}\right)=0$, a theorem of Donaldson [7] says that $\omega_{b}$ is in fact unique. For each $b$, there is a unique function $\phi_{b} \in C^{\infty}\left(Y_{b}\right)$ with mean-value zero (with respect to $\left.\omega_{b}\right)$ and such that $\left.\omega^{\prime}\right|_{Y_{b}}+i \bar{\partial} \partial \phi_{b}=\omega_{b}$. Provided the $\phi_{b}$ are smooth in $b$, they fit together to give a function $\phi \in C^{\infty}(X)$; then $\omega^{\prime \prime}=\omega^{\prime}+i \bar{\partial} \partial \phi$ is a $(1,1)$-form in $\kappa_{r_{0}}$ whose fibrewise restriction is cscK. The smoothness of $\omega^{\prime \prime}$ is provided by the following lemma.

Lemma 2.1. The functions $\phi_{b}$ depend smoothly on $b$.

Proof. This is essentially a standard result in the theory of elliptic PDEs. Choose a local trivialisation for $\pi$ over some disc $D \subset B,\left.X\right|_{D} \cong D \times Y$ as smooth manifolds. By restriction in this trivialisation, the complex structure and Kähler form $\omega^{\prime}$ on $X$ give a smooth family of Kähler structures $\left(J_{b}, \omega_{b}^{\prime}\right)$ on $Y$ parameterised by $b \in D$.

Define a map $S: D \times C^{\infty}(Y) \rightarrow C^{\infty}(Y)$ by

$$
S(b, \phi)=\operatorname{Scal}\left(\omega_{b}^{\prime}+i(\bar{\partial} \partial)_{b} \phi\right)
$$

where $(\bar{\partial} \partial)_{b}$ is defined with respect to $J_{b}$. $S$ extends to a smooth map $D \times L_{k+4}^{2}(Y) \rightarrow$ $L_{k}^{2}(Y)$. ( $S$ is smooth in the $D$ factor because $\operatorname{Scal}(\omega, J)$ depends smoothly on $\omega$ and $J$, see, for example, section 2.2 in [9].)

By definition, $S\left(b, \phi_{b}\right)$ is a constant. The linearisation of $S$ with respect to $\phi$ at such a point is given by

$$
\mathcal{D}_{b}^{*} \mathcal{D}_{b}: C^{\infty}(Y) \rightarrow C^{\infty}(Y)
$$

Here $\mathcal{D}_{b}$ is defined by

$$
\mathcal{D}_{b}(\psi)=\bar{\partial}_{b}\left(h_{b}(\psi)\right)
$$

where $h_{b}(\psi)$ is the Hamiltonian vector field of $\psi$ with respect to $\omega_{b}^{\prime}$ and $\bar{\partial}_{b}$ is the $\bar{\partial}$ operator on $T Y$ determined by $J_{b}$. The map $\mathcal{D}_{b}^{*}$ is the $L^{2}$-adjoint of $\mathcal{D}$ with respect to the metric $\omega_{b}^{\prime}$. (The linearisation of the scalar curvature map is computed in several places in the literature; see, for example, [9].)

Now $\mathcal{D}_{b}^{*} \mathcal{D}_{b}$ is elliptic (again, see [9]), self adjoint and so has index zero. Since the fibres of $\pi$ have no non-zero holomorphic vector fields, $\operatorname{ker} \mathcal{D}_{b}^{*} \mathcal{D}_{b}$ is the constant functions. Hence $\mathcal{D}_{b}^{*} \mathcal{D}_{b}$ is an isomorphism modulo the constants. By the implicit function theorem, the map $b \mapsto \phi_{b}$ is a smooth map $D \rightarrow L_{k}^{2}(Y)$ for any $k$. By 
Sobolev embedding, it is a smooth map $D \rightarrow C^{r}(Y)$ for any $r$. Hence $\phi_{b}$ is smooth in $b$.

Now $\omega^{\prime \prime}=\omega^{\prime}+i \bar{\partial} \partial \phi$ needn't be Kähler as it may be degenerate transverse to the fibres. To fix this, take a Kähler form $\omega_{B} \in \kappa_{B}$; for sufficiently large $r$, the form $\omega=\omega^{\prime \prime}+\left(r-r_{0}\right) \pi^{*} \omega_{B}$ is a Kähler metric in $\kappa_{r}$ whose fibrewise restriction is cscK.

The idea is that for large $r$, the geometry is dominated by that of the fibres which are cscK. One might hope then to be able to perturb the metric $\omega$ to a genuine $\operatorname{cscK}$ metric. A previous paper [9] considers this problem in detail. In the case where $X$ is a surface it solves it completely; in higher dimensions it reduces it to solving a certain $\mathrm{PDE}$ for a metric on $B$. To describe this PDE, some notation is required.

The fibrewise cscK metrics define a Hermitian structure in the vertical tangent bundle $V$ and hence in the relative canonical bundle $K_{X / B}=\Lambda^{\max } V^{*}$; denote its curvature by $F$. Notice that the restriction to a fibre of $-i F$ is the Ricci form of that fibre with its cscK metric.

The metric $\omega$ defines a vertical-horizontal decomposition of $T X$. Let $F_{H}$ denote the horizontal-horizontal component of $F$ with respect to this splitting. Define a form $a \in \Omega^{1,1}(B)$ by taking the fibrewise mean value of $i F_{H}$ with respect to $\omega$. More precisely, the differential $D \pi$ identifies the horizontal distribution restricted to $Y_{b}$ with $Y_{b} \times T_{b} B$; using this identification, on $Y_{b}, F_{H}: Y_{b} \rightarrow \Lambda^{1,1} T_{b}^{*} B$ is a vector valued function; then $a_{b} \in \Lambda^{1,1} T_{b}^{*} B$ is given by

$$
a_{b}=\frac{\int_{Y_{b}} i F_{H} \omega_{b}^{n}}{\int_{Y_{b}} \omega_{b}^{n}} .
$$

Theorem $2.2([9])$. Let $\pi: X \rightarrow B$ be a fibration and $L \rightarrow X$ a relatively ample line bundle as above. Suppose, moreover, that there is a Kähler metric $\omega_{B}$ on the base solving

$$
\operatorname{Scal}\left(\omega_{B}\right)-\operatorname{tr}_{\omega_{B}} a=\lambda,
$$

for some constant $\lambda$, and that there are no other cohomologous solutions to this equation. Then, for all sufficiently large $r$, the class $\kappa_{r}=\kappa+r \pi^{*}\left[\omega_{B}\right]$ contains a cscK metric.

The difficulty with applying Theorem 2.2 is that the PDE for $\omega_{B}$ is as awkward to solve as the cscK equation. However, the un-traced version,

$$
\rho\left(\omega_{B}\right)-a=\lambda \omega_{B},
$$

(where $\rho(\omega)$ denotes the Ricci form of $\omega$ ) is essentially the complex Monge-Ampere equation. When $\lambda \leq 0$ this has been solved by Aubin [3] and Yau [20]. Before describing this, it is first necessary to give a cleaner description of $a$. (See (1.1) and (1.2) for the definitions of $s$ and $\alpha$.)

Lemma 2.3.

$$
a=\frac{1}{\pi_{*}\left(\omega^{n}\right)} \pi_{*}\left[\left(i F+\frac{2 \pi s}{n+1} \omega\right) \wedge \omega^{n}\right]
$$

In particular, $a$ is closed and $[a]=2 \pi \alpha$. 
Proof. Write $F_{V}$ and $F_{H}$ for the purely vertical and purely horizontal components of $F$ respectively. Since $-i F_{V}$ is the Ricci-form of $\omega_{b}$ which is $\operatorname{cscK},-n i F_{V} \wedge \omega_{b}^{n-1}=$ $2 \pi s \omega_{b}^{n}$. So,

$$
\begin{aligned}
\pi_{*}\left(i F_{V} \wedge \omega^{n}\right) & =\pi_{*}\left(n i F_{V} \wedge \omega_{b}^{n-1} \wedge \omega_{H}\right) \\
& =-2 \pi s \pi_{*}\left(\omega_{b}^{n} \wedge \omega_{H}\right) \\
& =-\frac{2 \pi s}{n+1} \pi_{*}\left(\omega^{n+1}\right)
\end{aligned}
$$

Hence,

$$
\begin{aligned}
\pi_{*}\left(i F \wedge \omega^{n}\right) & =\pi_{*}\left(i F_{H} \wedge \omega^{n}\right)+\pi_{*}\left(i F_{V} \wedge \omega^{n}\right) \\
& =\pi_{*}\left(\omega^{n}\right) a-\frac{2 \pi s}{n+1} \pi_{*}\left(\omega^{n+1}\right)
\end{aligned}
$$

which gives the formula for $a$.

To deduce that $[a]=2 \pi \alpha$, observe that $[i F]=2 \pi c_{1}\left(K_{X / B}\right)$, whilst $[\omega]=\kappa_{r}=$ $c_{1}(L)+\pi^{*} \kappa_{B}$. Twisting $L$ by bundles pulled back from $B$ leaves $\alpha$ unchanged, so $\alpha$ can be computed using $\kappa_{r}$ in place of $c_{1}(L)$.

Everything is now in place to complete the proof.

Proof of Theorem 1.1. Let $\omega_{B}^{\prime} \in \kappa_{B}$ be a Kähler metric on $B$. Given $\phi \in C^{\infty}(B)$, let $\omega_{B}=\omega_{B}^{\prime}+i \bar{\partial} \partial \phi$. The aim is to find $\phi$ such that $\omega_{B}$ solves equation (2.2). The reduction to the complex Monge-Ampere equation is standard; brief details are given below, for more information see, for example, the account in [12].

Since $\rho\left(\omega_{B}^{\prime}\right)-a \in 2 \pi\left(c_{1}(B)-\alpha\right)$, there exists $f$ such that

$$
\rho\left(\omega_{B}^{\prime}\right)-a=\lambda \omega_{B}^{\prime}+i \bar{\partial} \partial f
$$

where $\lambda=0$ if $a-c_{1}(B)=0$ and $\lambda=-2 \pi$ if $a-c_{1}(B)>0$. Define $M(\phi)=\omega_{B}^{r} / \omega_{B}^{\prime r}$ where $r=\operatorname{dim} B$. Then $\rho\left(\omega_{B}\right)=\rho\left(\omega_{B}^{\prime}\right)+i \bar{\partial} \partial \log M(\phi)$. Hence $\omega_{B}$ solves (2.2) if and only if $\phi$ solves $\log M(\phi)=\lambda \phi-f$. By the famous theorems of Aubin [3] $(\lambda<0)$ and Yau [20] $(\lambda=0)$, this has a unique solution. Hence there is a unique solution $\omega_{B}$ to $(2.2)$ in $\left[\omega_{B}^{\prime}\right]$.

It remains to show that $\omega_{B}$ is the unique solution in $\left[\omega_{B}^{\prime}\right]$ to the traced equation (2.1) appearing in Theorem 2.2. Suppose $\omega_{B}$ solves Scal $-\operatorname{tr} a=\lambda$, with $\lambda \omega_{B} \in a-c_{1}(B)$. Now $\rho\left(\omega_{B}\right)-a$ is $\bar{\partial}$-closed and it follows from the Kähler identity $[\operatorname{tr}, \partial]=i \bar{\partial}^{*}$ and the fact that Scal $-\operatorname{tr} a$ is constant that it is also $\bar{\partial}^{*}$-closed. Hence $\rho\left(\omega_{B}\right)-a$ and $\lambda \omega_{B}$ are harmonic representatives for the same class and so, by Hodge theory, are equal. That is, $\omega_{B}$ solves $(2.2)$ also.

Theorem 1.1 now follows from Theorem 2.2.

\section{Non-negativity of the CM-line bundle}

This section gives the proof of Theorem 1.2. The key step is provided by the following theorem of Cornalba-Harris [5].

Theorem 3.1 (Cornalba-Harris). Let $\pi: X \rightarrow B$ be a flat proper morphism of varieties where $B$ is one-dimensional and $X$ is $(n+1)$-dimensional. Let $E \rightarrow X$ be a relatively very ample line bundle whose restriction to at least one fibre gives a Hilbert semi-stable embedding. 
Then

$$
h c_{1}(E)^{n+1}-(n+1) c_{1}(E)^{n} \pi^{*} c_{1}\left(\pi_{*} E\right) \geq 0
$$

where $h$ is the rank of $\pi_{*} E$.

Theorem 1.2 is proved by looking at the asymptotics of this result.

Proof of Theorem 1.2. Recall that $\pi: X \rightarrow B$ is a flat proper morphism of varieties, $L$ is a relatively ample line bundle whose restriction to a generic fibre is asymptotically semi-stable. The aim is to show that $\alpha$ (defined in (1.2)) is nef. It suffices to consider the case when $B$ is a curve.

For sufficiently large $m, L^{m}$ is relatively very ample and makes at least one of the fibres Hilbert semi-stable. By Theorem 3.1, with $E=L^{m}$,

$$
h(m) m^{n+1} c_{1}(L)^{n+1}-m^{n}(n+1) c_{1}(L)^{n} \pi^{*} c_{1}\left(\pi_{*}\left(L^{m}\right)\right) \geq 0,
$$

where $h(m)=\operatorname{rank} \pi_{*}\left(L^{m}\right)$ is the Hilbert polynomial of the restriction of $L$ to a fibre.

By Grothendieck-Riemann-Roch,

$$
c_{1}\left(\pi_{*} L^{m}\right)=\pi_{*}\left(\frac{c_{1}(L)^{n+1}}{(n+1) !} m^{n+1}-\frac{c_{1}(L)^{n} c_{1}\left(K_{X / B}\right)}{2 n !} m^{n}+\cdots\right) .
$$

On the other hand,

$$
h(m)=\pi_{*}\left(c_{1}(L)^{n}\right)\left(\frac{1}{n !} m^{n}+\frac{s}{2 n !} m^{n-1}+\cdots\right) .
$$

Apply $\pi_{*}$ to (3.1) and collect terms. The $m^{2 n+1}$-coefficient is zero and so the leading term is $m^{2 n}$. The coefficient of $m^{2 n}$ is $\alpha /(2(n+1) !)$. Since $\pi_{*}$ of (3.1) is positive for all large $m$, its leading coefficient must be positive.

One situation in which the positivity of $c_{1}\left(L_{\mathrm{CM}}\right)$ is completely understood is that of curves of genus at least two. In this case, all polarisations are multiples of the canonical polarisation and $c_{1}(\mathrm{CM})$ is essentially $\pi_{*}\left(c_{1}\left(K_{X / B}\right)^{2}\right)$, the first tautological class, whose positivity properties are completely known. It is not ample on the Hilbert scheme, even over the stable locus ([11], page 313). On the other hand, it is ample when considered over the smooth locus ([11], page 312).

This tallies with the higher dimensional result of Fujiki-Schumacher [10], which shows that, provided the fibres have no holomorphic vector fields, $c_{1}\left(L_{\mathrm{CM}}\right)$ is ample over the part of the smooth locus which admits a cscK metric. With this in mind, it seems reasonable to guess that $c_{1}\left(L_{\mathrm{CM}}\right)$ is ample on the smooth, asymptotically semi-stable locus of the Hilbert scheme.

Another question is whether or not the stability hypothesis in Theorem 1.2 is necessary. Certainly some stability hypothesis is required. In [5] Cornalba and Harris discuss a family of Hilbert unstable varieties for which their inequality is violated. Is it possible, however, to replace asymptotic Hilbert semi-stability with K-semi-stability?

\section{Examples}

A large class of submersions satisfying the hypotheses of Corollary 1.3 are provided by the iterated surface bundles of Morita. Their construction is given in detail in chapter 4 of [14]. 
In what follows, a $\Sigma_{g}$-bundle is a holomorphic submersion between complex manifolds whose fibres are curves of genus $g$. For each positive integer $k$, define the set $\mathcal{C}_{k}$ of connected compact complex $k$-folds recursively as follows: the only element of $\mathcal{C}_{0}$ is a single point; in general, $\mathcal{C}_{k+1}$ is defined to be the set consisting of any finite covering of the total space of a $\Sigma_{g}$-bundle with $g \geq 2$ and whose base belongs to $\mathcal{C}_{k}$. Let $\mathcal{C}=\bigcup \mathcal{C}_{k}$ and call its members iterated surface bundles.

Given a $\Sigma_{g}$-bundle $\pi: X \rightarrow B$ with $g \geq 2$ whose total space is an iterated surface bundle, Morita produces a finite covering $B^{\prime} \rightarrow X$, and a $\Sigma_{g^{\prime}}$-bundle $X^{\prime} \rightarrow B^{\prime}$ where $g^{\prime}=m^{2} g-\frac{1}{2} m(m+1)+1$ is again at least 2. Moreover, the moduli of the fibres of $X^{\prime}$ are not constant. (In [14], Morita shows that certain characteristic classes of surface bundles are non-zero on $X^{\prime}$; these classes vanish on isotrivial families.) It remains to check that these fibrations satisfy the hypotheses of Corollary 1.3.

If $\pi: X \rightarrow B$ is a non-constant family of smooth curves of genus at least 2 , then the relative canonical bundle $K_{X / B}$ is ample (see [11], page 309). If the canonical bundle of $B$ is also ample then so is the canonical bundle of $X$. That is, if $c_{1}(B)<0$ then $c_{1}(X)<0$.

The short exact sequence $0 \rightarrow V \rightarrow T X \rightarrow \pi^{*} T B \rightarrow 0$, where $V$ denotes the veritcal tangent bundle, gives a long exact sequence in cohomology $0 \rightarrow H^{0}(X, V) \rightarrow$ $H^{0}(X, T X) \rightarrow H^{0}\left(X, \pi^{*} T B\right) \rightarrow \cdots$. Now $H^{0}(X, V)=0$ as the fibres admit no nonzero holomorphic vector fields, whilst $H^{0}\left(X, \pi^{*} T B\right)=H^{0}\left(B, \pi_{*} \pi^{*} T B\right)=H^{0}(B, T B)$, so $H^{0}(B, T B)=0$ implies that $H^{0}(X, T X)=0$. Similarly, finite covers of $B$ have no holomorphic vector fields.

Therefore repeatedly applying Morita's construction for various choices of $m$ and $g$ to $\Sigma_{g}$-bundles over a point produces infinitely many Kähler manifolds $X$ of arbitrary dimension all of which satisfy the hypotheses of Corollary 1.3.

It should be pointed out that there are other ways of producing infinitely many cscK manifolds in each dimension, the most obvious being products. Kähler-Einstein metrics give non-trivial examples. For examples which are cscK but not KählerEinstein, the Kähler-Einstein metrics can be deformed via a theorem of LeBrun and Simanca [13] which says that, in the absence of holomorphic vector fields, if a Kähler class admits a cscK representative, then nearby Kähler classes also admit a cscK representative. Alternatively, a result of Arezzo and Pacard [2] states that, in the absence of holomorphic vector fields, a cscK metric on $X$ gives a cscK metric on the blow-up of $X$ at a point, providing yet more examples. All these approaches, however, ultimately rely on deforming Kähler-Einstein metrics. By contrast, the examples produced via Theorem 1.1 lie in classes arbitrarily far from the canonical class and are unrelated to any Kähler-Einstein metric on $X$ or $B$. 


\section{References}

[1] V. Apostolov, D. M. J. Calderbank, P. Gauduchon, and C. W. Tonnesen-Friedman, Hamiltonian 2-forms in kähler geometry, 3 compact examples, math.DG/0501516, 2005.

[2] C. Arezzo and F. Pacard, Blowing up and desingularizing kähler orbifolds of constant scalar curvature. math.DG/0411522, 2004.

[3] T. Aubin, Équations du type Monge-Ampère sur les variétés kählériennes compactes, Bull. Sci. Math. (2) 102 (1978), 63-95.

[4] E. Calabi, Extremal Kähler metrics, Seminar on Differential Geometry, Ann. of Math. Stud., Princeton Univ. Press, Princeton, N.J., 1982, 259-290.

[5] M. Cornalba and J. Harris, Divisor classes associated to families of stable varieties, with applications to the moduli space of curves, Ann. Sci. École Norm. Sup. (4) 21 (1988), no. 3, 455-475.

[6] S. K. Donaldson, Remarks on gauge theory, complex geometry and 4-manifold topology, Fields Medalists' Lectures, 384-403, World Sci. Publ., N.J., 1997.

[7] Scalar curvature and projective embeddings. I, J. Differential Geom. 59 (2001), no.3, $479-522$.

[8] _ Scalar curvature and stability of toric varieties, J. Differential Geom. 62 (2002), no. 2, 289-349.

[9] J. Fine, Constant scalar curvature Kähler metrics on fibred complex surfaces, J. Differential Geom. 68 (2004), no.3, 397-432.

[10] Akira Fujiki and Georg Schumacher, The moduli space of extremal compact Kähler manifolds and generalized Weil-Petersson metrics, Publ. Res. Inst. Math. Sci. 26 (1990), no. 1, 101-183.

[11] J. Harris and I. Morrison, Moduli of curves, Graduate Texts in Mathematics 187 SpringerVerlag, New York, 1998.

[12] D. D. Joyce, Compact manifolds with special holonomy, Oxford Mathematical Monographs, Oxford University Press, Oxford, 2000.

[13] C. LeBrun and S. R. Simanca, Extremal Kähler metrics and complex deformation theory, Geom. Funct. Anal. 4 (1994), no. 3, 298-336.

[14] S. Morita. Geometry of characteristic classes, Translations of Mathematical Monographs, American Mathematical Society, Providence, RI, 2001.

[15] S. T. Paul and G. Tian, Algebraic and analytic k-stability, math.DG/0405530, 2004.

[16] _ Analysis of geometric stability, Int. Math. Res. Not., 2004, 2555-2591.

[17] Y. Rollin and M. A. Singer. Construction of kähler surfaces with constant scalar curvature, math.DG/0412405, 2004.

[18] G. Tian, Kähler-Einstein metrics with positive scalar curvature, Invent. Math. 130 (1997), no. $1,1-37$.

[19] , The K-energy on hypersurfaces and stability, Comm. Anal. Geom. 2 (1994), no. 2, 239-265.

[20] S.-T. Yau. On the Ricci curvature of a compact Kähler manifold and the complex Monge-Ampère equation. I, Comm. Pure Appl. Math 31 (1978), no. 3, 339-411.

[21] _ Open problems in geometry, Differential geometry: partial differential equations on manifolds (Los Angeles, CA, 1990), Proc. Sympos. Pure Math. 54 Part 1, Amer. Math. Soc., Providence, RI, 1993, 1-28.

Mathematics Department., Imperial College., 180 Queen's Gate, London, SW7 2AZ, UK.

E-mail address: joel.fine@imperial.ac.uk 